\title{
Regional homogeneity in cognition with frontal lobe injury-A resting-state fMRI study
}

\author{
Yang Liu ${ }^{1}$, Zheng Ding ${ }^{2}$, Xiao-yang Wang ${ }^{3}$, Su-jian Zhou ${ }^{4}$, Hui Xiao $^{3}$, Xiao-xin Lu ${ }^{4}$ and Hui-ping Peng ${ }^{4 *}$ \\ ${ }^{1}$ Department of Rehabilitation Medicine, The Second Affiliated Hospital of Nanchang University, Nanchang, Jiangxi, 330000, China \\ ${ }^{2}$ Department of Rehabilitation Medicine, Xiangtan city central hospital, Xiangtan, Hunan, 411100, China \\ ${ }^{3}$ Department of Media Imaging, Fuzhou general hospital. Fuzhou, Fujian, 350025, China \\ ${ }^{4}$ Department of Rehabilitation Medicine, Fuzhou General Hospital. Fuzhou, Fujian, 350025, China
}

\begin{abstract}
Objective: To investigate the spontaneous brain activity changes with the cognitive disorders of traumatic brain injury in the frontal lobe using resting-state fMRI and regional homogeneity ( $\mathrm{ReHo})$.

Methods: Thirteen frontal lobe injuries with cognition impairment were sampled as traumatic brain injury group (TBIs) and fourteen healthy persons as the normal control group(NCs). General cognition was assessed through Mini-Mental State Examination(MMSE). Resting-state fMRI and T1-weighted imaging data were then collected. The ReHo maps were obtained from resting-state fMRI, and two-sample t-test was performed between the two groups. Finally, pearson correlation analysis was conducted between ReHo values extracted from different brain regions and MMSE scores of the patients.

Results: Compared with the NCs group, the TBI group showed significantly increased ReHo in the left superior frontal gyrus, right middle occipital, right declive $(p<0.05)$ and significantly decreased ReHo in the left media frontal gyrus, left anterior cingulate, inferior frontal gyrus, right superior temporal, right supramarginal gyrus, right hippocampus/parahippocampal, left/right supplement motor area, right uvula $(p<0.05)$. In addition, a positive correlation was found between the ReHo index of the left superior frontal gyrus and the MMSE scores across all patients with TBI.

Conclusions: The ReHo method may provide an objective biomarker for evaluating the functional abnormity of the cognition in frontal lobe injuries. The cognitive disorders may be related to abnormal brain activity, and the imbalance of the neural network caused by the abnormal spontaneous neuronal activity may underlie the potential cognitive damage in the frontal lobe injury.
\end{abstract}

\section{Introduction}

Traumatic brain injury (TBI) is an important public health problem, featuring high morbidity rate, disability rate and mortality rate [1], which therefore contributes to considerable social and economic costs [2]. Clinically, most TBIs result in damage to frontal lobe areas due to its anatomical features, and affect frontal lobe-dependent functions $[3,4]$. As we all know, the frontal lobe is responsible for several cognitive function including attention, cognitive control, long-term memory, working memory, and especial in executive function [5-7]. What's more, executive dysfunction is strongly related to cognitive abilities included in planning, starting, carrying out, and monitoring complex behaviours that require attention, concentration, selectiveness of stimuli, abstract thinking, cognitive flexibility, self-control, and memory [8]. And all of the frontal lobe-dependent functions enable us to transform thoughts into complex behaviours, to initiate or inhibit events [9], to reflect on other people's mental states [10], and to plan a specific approach to each objective [11]. Therefore, the deficits in frontal lobe injuries can influence cognitive functions to the extent that social and occupational impairments may happen to patients [9]. It will result in difficulties for their emotional control, social adaptation, and even the health-related quality of life [12].

In the early 1990s, functional magnetic resonance imaging (fMRI) became popular in detecting difference in magnetic susceptibility between oxygenated and deoxygenated haemoglobin which could be used as an index of local brain activity [13]. These techniques could eventually be helpful in developing imaging biomarkers of cognitive and neurobehavioral sequelae, as well as predicting outcomes and prognosis [14]. However, the application of fMRI in clinical areas has been limited due to the complex data processing and the unsteadiness of technology. Resting-state fMRI (rs-fMRI) refers to a function magnetic resonance imaging which happens when participants are conducting none intentional thinking activity, with their eyes closed, being relaxed and still. Regional homogeneity ( $\mathrm{ReHo}$ ), as an analytic method based on Kendall's coefficient concordance (KCC), can be used to reflect synchronicity of regional local time sequence and to observe local consistency of neuron activity in the brain. Previous studies have firmly suggested that re-fMRI with ReHo play a crucial role in the pathophysiology of mTBI [15]. In addition, it has been reported that the amplitude of low-frequency fluctuation (ALFF, another analysis method for re-fMRI) in the cingulate gyrus was significantly positively related to working memory index (WMI) in the mild patient with a

${ }^{\star}$ Correspondence to: Hui-ping Peng, The office of Rehabilitation Medicine department, Fuzhou General hospital, 156 Xierhuan North Road, Fuzhou, Fujian, China, Tel: +86-13600823060, E-mail: 361140072@qq.com

Key words: Traumatic brain injury, frontal lobe, cognitive disorders, fMRI, Regional Homogeneity

Received: May 14, 2018; Accepted: May 28, 2018; Published: June 05, 2018 
TBI [16]. It is shown through literature review that, there was no study on the patients of TBI in frontal lobe. To further recapitulate frontal lobe injuries observed in cognition, we applied resting-state fMRI with ReHo to investigate the spontaneous brain activity changes.

\section{Materials and methods}

During April 2015 and July 2016, 13 patients (10 male and 3 females; mean age $\pm S D$ is $35.14 \pm 6.20$ years old) with TBI were recruited from Fuzhou General Hospital of Nanjing Military Command, PLA. Fuzhou, Fujian, China. All the patients had a history of brief coma of 13 to 15 points as assessed by the Glasgow coma scale (GCS). Conventional computed tomography (CT) or MRI examinations showed only frontal lobe injury, excluding previous disease history: brain trauma, surgery, cerebral tumour or other brain lesions. During the post-injury recovery stage, which lasted less than three mouths, all the patients continued to complain of headache, impaired concentration and cognitive impairment (MMSE scores < 27). All the recruited patients had not suffered mental symptom, whose index of Beck's Depression Inventory Scale (BDI) was less than 14 scores. Additionally, patients with epilepsy, patients with severe heart, liver, or renal dysfunction, patients with blood disorders, and patients with serious words, vision, hearing impairment were excluded. Fourteen (14) healthy subjects were recruited as control group. They were matched to the 13 patients with TBI with respect to age, gender, and education level. The control group included 10 males and 4 females, ranging from 18 to 55 years old (average, $33.83 \pm 6.65$ years old).

\section{Clinical assessment and image acquisition}

Clinical assessment was performed within 3 days before MR imaging for both patients with TBI and NCs, including GCS, MMSE and BDI. The BDI provided estimates of depressive symptoms.

A Siemens 3.0T superconductive MR scanner (Skyra, Siemens Medical, Germany) with a 12-channel phased-array head coil was used. The subjects from both the experimental group and control group received cranial MRI in the supine position, with their heads entering first. Anatomical imaging was performed using conventional MRI SE sequence scanning (T2WI, T2-FLAIR) and gradient-echo T1-weighted scanning to obtain whole-brain MRI anatomical images. Gradient-echo T1-weighted 3D anatomical image scanning (TR/TE $=1900 / 2.26 \mathrm{~ms}$, FOV $=256 \mathrm{~mm} \times 256 \mathrm{~mm}$, Flip angle $=9^{\circ}$, Slice thickness $=1.0 \mathrm{~mm}, \mathrm{Gap}=0$, Matrix $=256 \times 256$, Slice $=176$, Voxel size $=1 \mathrm{~mm} \times 1 \mathrm{~mm} \times 1 \mathrm{~mm}$.) was used for the Talairach transformation of the images and to visualize the morphology of brain structures.

Resting-state echo-planar imaging was accomplished through a single-shot gradient-echo weighted sequence to obtain BOLD brain signals $(\mathrm{TR} / \mathrm{TE}=2000 / 30 \mathrm{~ms}, \mathrm{FOV}=240 \mathrm{~mm} \times 240 \mathrm{~mm}$, Flip angle $=$ $90^{\circ}$, Slice thickness $=4.0 \mathrm{~mm}, \mathrm{Gap}=0.8 \mathrm{~mm}$, Matrix $=64 \times 64$, Slice $=$ 33 , Voxel size $=3.8 \mathrm{~mm} \times 3.8 \mathrm{~mm} \times 4.0 \mathrm{~mm}$ ). The subjects were asked to close their eyes and lie down, relax, stay awake, and try not to think about anything. The scanning process lasted for 8 minutes.

\section{Data analysis}

The pre-processing of data was carried out by using Data Processing Assistant for Resting-State fMRI (DPARSF) (Yan \& Zang, 2010, http://rfmri.org/DPARSF), which is based on Statistical Parametric Mapping (SPM8) (http://www.fil.ion.ucl.ac.uk/spm). The first 10 time points were deleted for magnetization equilibrium and adaptation of participants in the scanning environment, leaving 230 time points for further analysis. Then time correction was carried out to ensure that scanning layers obtained at different time points were corrected to be the obtained a same time point. The correction of head motion was then carried out. Participants who had more than $2 \mathrm{~mm}$ of translation or greater than $2^{\circ}$ rotation in any direction during the whole fMRI scan were excluded. Next, the functional images were spatially normalized to the Montreal Neurological Institute (MNI) space with a resampling voxel size of $3 \times 3 \times 3 \mathrm{~mm}^{3}$. Linear trends were removed, and a temporal band-pass filter $(0.01-0.08 \mathrm{~Hz})$ was applied to reduce the effects of lowfrequency drifts and high-frequency respiratory and cardiac noise for further ReHo analysis.

ReHo was calculated using REST 1.8 (State Key Laboratory of Cognitive Neuroscience and Learning Beijing Normal University). Viewer of REST1.8 was used to compare the voxel, peak value, MNI Coordinates between groups.

\section{Statistical analysis}

The SPSS 20.0 software package was employed to analyse participants' age, educational qualification and MMSE scores, the results of which were calculated as " $\mathrm{x} \pm \mathrm{s}$ ". Two-sample t-tests were performed to explore the differences in age and duration of education between TBIs and NCs, and a p-value of less than 0.05 (two-tailed) was considered to be statistically significant. For ReHo, we compared the voxel-wise differences between groups through two-sample t-test, and the statistical analysis was asked by a map. Voxels with a $\mathrm{p}$ value of less than 0.05 and a cluster size of over $78 \mathrm{~mm}^{3}$ (AlphaSim corrected) demonstrated a significant difference between two groups. REST 1.8 software package was used to extract ReHo values of the brain regions which showed differences. Pearson's correlation analysis was conducted to compare the ReHo values of different brain regions and MMSE scores.

\section{Results}

\section{Demographic and clinical data}

Demographic and clinical data are showed in table 1. Subjects from the TBIs and NCs showed no significant difference in age and years of education. All the recruited patients showed cognitive deficiency as their MMSE scores were less than 27. Besides, healthy subjects had normal MMSE scores (> 27), and patients from the TBIs had significantly higher levels of cognition impairment than healthy subjects from the NCs $(p<0.001)$.

\section{ReHo analysis}

Compared with NCs, patients in the TBIs showed significantly increased ReHo in the left superior frontal gyrus and right middle occipital, right declive (Figure 1 and Table 2) and decreased ReHo in the left media frontal gyrus, left anterior cingulate, left inferior frontal gyrus, right superior temporal, right supramarginal gyrus, right hippocampus/parahippocampal, left/right supplement motor area, right uvula (Figure 2 and Table 3 ).

\section{Correlation analysis}

A positive correlation was found between the ReHo of the left superior frontal gyrus and MMSE scores $(\mathrm{R} 2=0.54, p<0.001)$ (Figure 3 ).

\section{Discussion}

Currently, there has been a lack of research on the recognition of TBI models in the investigation of frontal lobe injuries despite the prevalence of focal contusions to the frontal lobe in patient with a TBI $[17,18]$. In our study, we recruited cognitive disorders in frontal 
Table 1. Demographic and Clinical Features between TBIs and NCs

\begin{tabular}{|c|c|c|c|c|c|c|}
\hline \multirow{2}{*}{ Groups } & \multirow{2}{*}{ Cases } & \multicolumn{2}{|c|}{ Gender } & \multirow{2}{*}{$\begin{array}{l}\text { Age } \\
(y r)\end{array}$} & \multirow{2}{*}{$\begin{array}{c}\text { Education } \\
\quad(\mathrm{yr})\end{array}$} & \multirow{2}{*}{ MMSE } \\
\hline & & male & Female & & & \\
\hline TBIs & 13 & 10 & 3 & $35.14 \pm 6.20$ & $8.86 \pm 2.12$ & $20.62 \pm 3.33$ \\
\hline NCs & 14 & 10 & 4 & $33.83 \pm 6.65$ & $9.20 \pm 3.42$ & $28.40 \pm 1.14$ \\
\hline $\mathrm{t}$ & & & & 0.37 & -0.22 & -5.03 \\
\hline$P$ & & & & 0.72 & 0.83 & 0.00 \\
\hline
\end{tabular}

Data are presented as mean \pm standard deviation or number; a $p$-value less than 0.05 (two-tailed) was considered to be statistically significant; a $p$-value more than 0.05 (two-tailed) was considered to be no statistically significant

Table 2. Brain Regions showing significant differences in enhanced ReHo between TBIs and NCs

\begin{tabular}{|c|c|c|c|c|c|c|c|c|}
\hline \multirow{2}{*}{ Brain region } & \multirow{2}{*}{$\mathrm{L} / \mathrm{R}$} & \multicolumn{3}{|c|}{ MNI Coordinates } & \multirow{2}{*}{ voxels } & \multicolumn{2}{|c|}{ ReHo } & \multirow{2}{*}{$\mathrm{T}$} \\
\hline & & $\mathrm{X}$ & $\mathrm{Y}$ & $\mathrm{Z}$ & & TBIs & $\mathrm{NCs}$ & \\
\hline superior frontal gyrus & $\mathrm{L}$ & -6 & 57 & 36 & 127 & $1.00 \pm 0.28$ & $0.43 \pm 0.18$ & 5.37 \\
\hline middle occipital & $\mathrm{R}$ & 39 & -81 & 6 & 111 & $0.94 \pm 0.23$ & $0.40 \pm 0.29$ & 3.76 \\
\hline Declive & $\mathrm{R}$ & 15 & -90 & -27 & 98 & $0.64 \pm 0.22$ & $0.09 \pm 0.29$ & 3.99 \\
\hline
\end{tabular}

MNI, Montreal Neurological Institute; $\mathrm{x}, \mathrm{y}, \mathrm{z}$ coordinates of primary peak locations in the space of MNI; $\mathrm{t}$, statistical value of peak voxel; $p<0.05$, cluster size $>78$ mm ${ }^{3}$ corrected for AlphaSim multiple comparisons.

Table 3. Brain regions showing significant differences in decreased ReHo between TBIs and NCs

\begin{tabular}{|c|c|c|c|c|c|c|c|c|}
\hline \multirow{2}{*}{ Brain region } & \multirow{2}{*}{$\mathbf{L} / \mathbf{R}$} & \multicolumn{3}{|c|}{ MNI Coordinates } & \multirow{2}{*}{ voxels } & \multicolumn{2}{|c|}{ ReHo } & \multirow[t]{2}{*}{$\mathbf{T}$} \\
\hline & & $\mathrm{X}$ & $\mathrm{Y}$ & Z & & TBIs & $\mathrm{NCs}$ & \\
\hline $\begin{array}{l}\text { media frontal gyrus } \\
\text { and anterior cingulate }\end{array}$ & $\mathrm{L}$ & 9 & 48 & -9 & 123 & $0.41 \pm 0.34$ & $0.98 \pm 0.25$ & -3.54 \\
\hline inferior frontal gyrus & $\mathrm{L}$ & -51 & 39 & -3 & 78 & $0.45 \pm 0.95$ & $1.21 \pm 0.41$ & -3.74 \\
\hline $\begin{array}{l}\text { Hippocampus and } \\
\text { parahippocampal }\end{array}$ & $\mathrm{R}$ & 33 & -21 & -12 & 78 & $-0.63 \pm 0.20$ & $-0.32 \pm 0.30$ & -3.28 \\
\hline $\begin{array}{l}\text { superior temporal; supramarginal } \\
\text { gyrus }\end{array}$ & $\mathrm{R}$ & 66 & -34 & 24 & 153 & $-0.18 \pm 0.14$ & $0.29 \pm 0.16$ & -4.38 \\
\hline supplement motor area & $\mathrm{L} / \mathrm{R}$ & -9 & -3 & 69 & 142 & $-0.03 \pm 0.26$ & $0.52 \pm 0.26$ & -4.33 \\
\hline uvula & $\mathrm{L}$ & -3 & -69 & -39 & 118 & $-0.58 \pm 0.14$ & $-0.13 \pm 0.27$ & -3.96 \\
\hline
\end{tabular}

MNI, Montreal Neurological Institute; $\mathrm{x}, \mathrm{y}, \mathrm{z}$ coordinates of primary peak locations in the space of MNI; $\mathrm{t}$, statistical value of peak voxel; $p<0.05$, cluster size $>78$ mm ${ }^{3}$ corrected for AlphaSim multiple comparisons.

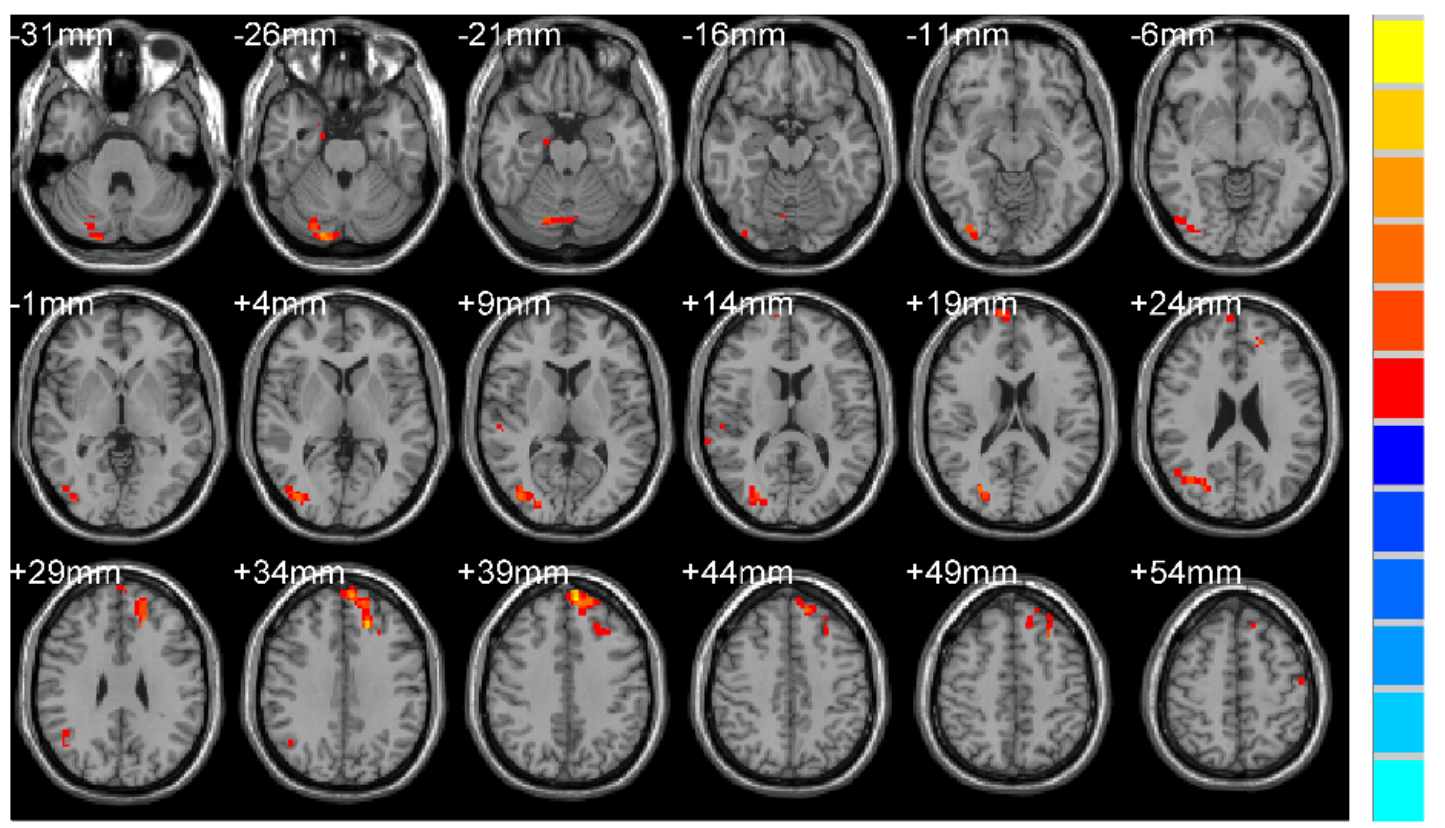

4.76

4.15

3.54

2.93

2.32

1.71

$-1.7$

$-2.2$

-2.8 .

$-3 \cdot 4$

$-3.9$

$-4 \cdot 5$

Figure 1. Compared with NCs, the enhanced brain region of ReHo, $p<0.05$, cluster size $>78 \mathrm{~mm}^{3}$ corrected for AlphaSim multiple comparisons. 


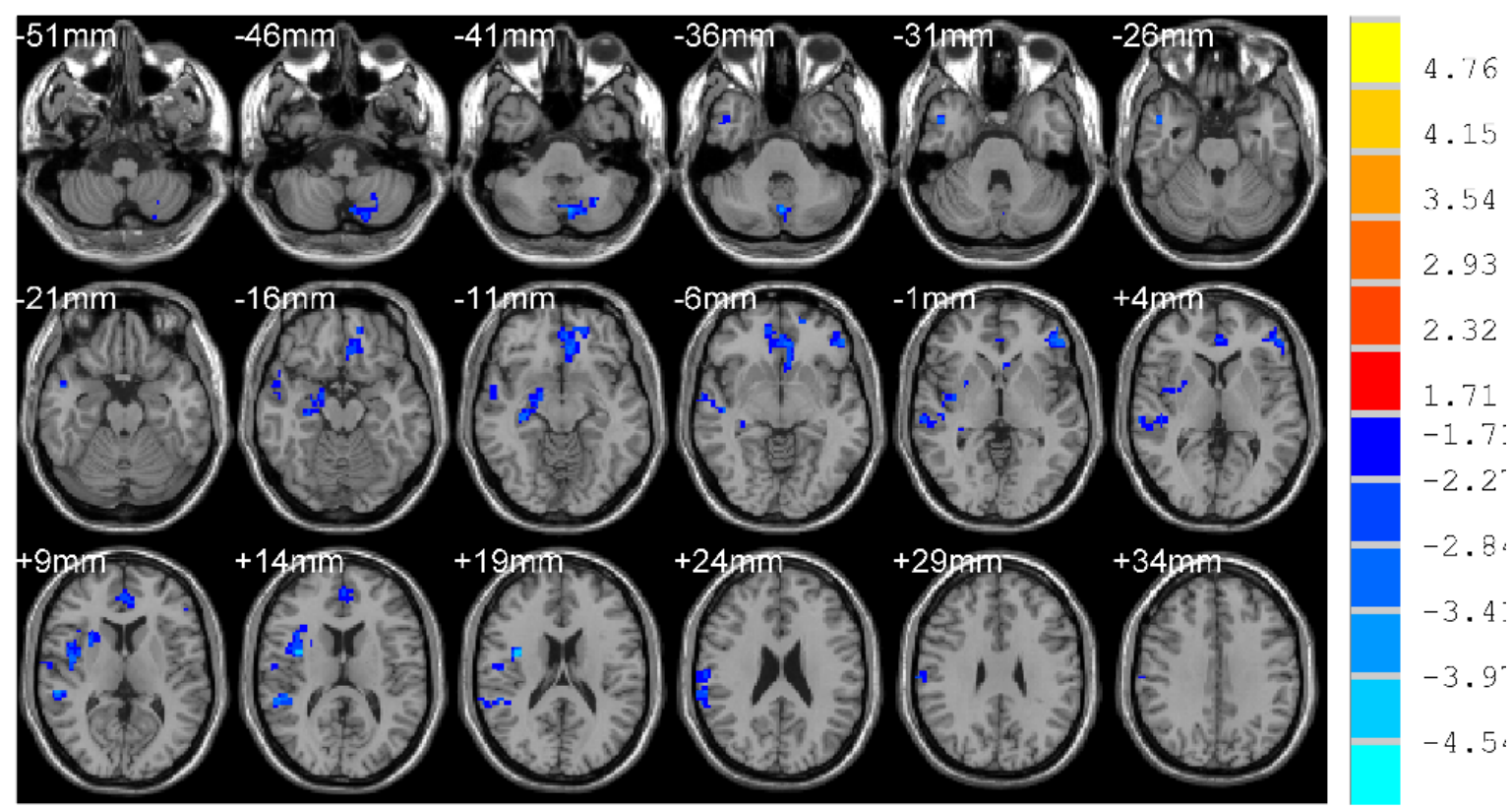

Figure 2. Compared with NCs, the decreased brain region of ReHo, $p<0.05$, cluster size $>78 \mathrm{~mm}^{3}$ corrected for AlphaSim multiple comparisons.

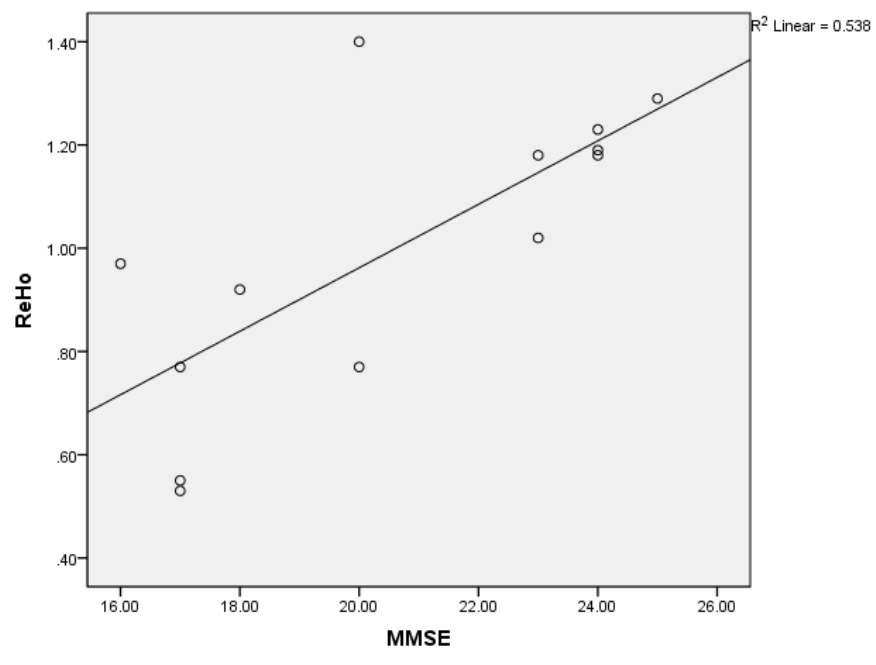

The Left Superior Frontal Gyrus

Figure 3. A positive correlation was found between the ReHo of the left superior frontal gyrus and MMSE scores.

lobe injuries by MRI examinations and we found that TBIs showed significantly increased ReHo in the left superior frontal gyrus, right middle occipital and decreased ReHo in the left media frontal gyrus, left anterior cingulate, left inferior frontal gyrus, right superior temporal, right supramarginal gyrus, right hippocampus/parahippocampal, left/ right supplement motor area and right uvula. The findings of decreased ReHo are partly consistent with Zhan's research [15].

In this paper, an important finding was increased ReHo in the left superior frontal gyrus and a positive correlation between the ReHo values and MMSE scores. These results suggest that the ReHo in superior frontal gyrus can respond to cognitive level to some extent in frontal lobe injuries, and the function of the frontal gyrus may show compensatory enhancement at the stage of cognitive impairment [19]. Olsen had also put forward that increased brain activations typically observed in survivors of TBI might represent injury-specific compensatory adaptations in everyday life situations. What's more, we think that the cognitive compensation will increase as long as the values of ReHo improve. Another finding was that the changes of spontaneous brain activity was identified to be related to a neural network, which consisted of the prefrontal cortex, premotor area, parietal lobe, anterior cingulate cortex, and supplementary motor area [20]. Moreover, Gillis [21] affirmed this neural network may influence the cognitive mechanisms of the TBIs. They designed fMRI tasks for the TBIs, which involved working memory encoding and maintenance. Those patients showed hyperactivation in the right dorsolateral prefrontal cortex, and this might be related to memory encoding.

In the present study, decreased ReHo in the left media frontal gyrus, left anterior cingulate, left inferior frontal gyrus, right hippocampus/ parahippocampal were observed. Takahashi suggest that hippocampus might affect the local hippocampal function and is thought to be important for cognitive functions such as memory, executive function and verbal fluency, as well as brain functions beyond the hippocampus such as the prefrontal cortex [22]. However, the hippocampus-frontal interaction mechanism is still unclear. A study of maladaptive response to stressful military service suggests that stress could lead to reduction in hippocampus volume and connectivity with the ventromedial prefrontal cortex together [23]. This indicates that the hippocampus and the prefrontal cortex have affected each other [24]. Using rigorous permutation testing to define human brain on structural-functional relationships, Harms found that the volume of the hippocampus was correlated with clusters in left inferior frontal gyrus and the dorsal anterior cingulate cortex [25]. However, we did not find ReHo values on those regions were correlated with the MMSE scores. Furthermore, previous fMRI studies have revealed that cortical volume reductions are bilaterally prominent in frontal, temporal, and inferior parietal regions in patient with a TBI and atrophy of the right anterior cingulate may contribute to reduced performance on cognition such as executive function tasks [26]. Becker et. al also demonstrated a modulation of the influence of the anterior cingulate cortex on the dorsolateral prefrontal cortex with novel task execution and they believe that the anterior cingulate cortex activity increased the dorsolateral prefrontal cortex 
[27]. Taking all together, the frontal lobe injuries may reduce the frontal gyrus, anterior cingulate cortex and hippocampus functions, which, in return, influenced cognitive function.

Our study also found that cerebellum is not limited to motor control [28] but is associated with cognitive function. Compared to the NCs, right declive in the TBIs was increased and right uvula was decreased. This may be related to hippocampal-cerebellar interactions which can functionally improve cognitive collaborations [29].

In conclusion, the ReHo method may provide an objective biomarker for evaluating the functional abnormity of the cognition in frontal lobe injuries. The cognitive disorders with frontal lobe injuries demonstrated abnormal spontaneous neuronal activities. And the imbalance of the neural network caused by the abnormal neuronal activity may underlie the potential cognitive damage.

This study has several limitations. First, the sample size was relatively small. A larger sample size is needed to increase the reliability in future studies. Second, cognitive assessment was incomplete, and the memory function and executive function will be further assessed. Third, the results of ReHo only reflect function in patients with TBI. In the future we will design both the Diffusion Tensor Imaging (DTI) and rs-fMRI to explore the relationship between structural and functional deficits.

\section{Acknowledgment}

This study was funded by the foundation of Program innovative team of Fuzhou General Hospital (2014CXTD08) and The Natural Science Fundation of Fujian Province (2016J0158).

\section{References}

1. Cuthbert JP, Harrison-Felix C, Corrigan JD, Kreider S, Bell JM, et al. (2015) Epidemiology of adults receiving acute inpatient rehabilitation for a primary diagnosis of traumatic brain injury in the United States. J Head Trauma Rehabil 30:122-135. [Crossref]

2. de Almeida CE, de Sousa Filho JL, Dourado JC, Gontijo PA, Dellaretti MA, et al. (2016) Traumatic Brain Injury Epidemiology in Brazil. World Neurosurg 87: 540-547. [Crossref]

3. Stuss DT (2011) Traumatic brain injury: relation to executive dysfunction and the frontal lobes. Curr Opin Neurol 24: 584-589. [Crossref]

4. van der Horn HJ, Liemburg EJ, Aleman A, Spikman JM, van der Naalt J (2016) Brain Networks Subserving Emotion Regulation and Adaptation after Mild Traumatic Brain Injury. J Neurotrauma 33:1-9. [Crossref]

5. Witt ST, Lovejoy DW, Pearlson GD, Stevens MC (2010) Decreased prefrontal cortex activity in mild traumatic brain injury during performance of an auditory oddball task. Brain Imaging Behav 4:232-247. [Crossref]

6. Kim C, Kroger JK, Calhoun VD, Clark VP (2015) The role of the frontopolar cortex in manipulation of integrated information in working memory. Neurosci Lett 595: 25-29. [Crossref]

7. Ghawami H, Sadeghi S, Raghibi M, Rahimi-Movaghar V (2017) Executive Functioning of Complicated-Mild to Moderate Traumatic Brain Injury Patients with Frontal Contusions. Appl Neuropsychol Adult 24: 299-307. [Crossref]

8. Rabinovici GD, Stephens ML, Possin KL (2015) Executive dysfunction. Continuum (Minneap Minn) 21: 646-659. [Crossref]

9. Jackson WM, Davis N, Sands SA, Whittington RA, Sun LS (2016) Physical Activity and Cognitive Development: A Meta-Analysis. J Neurosurg Anesthesiol 28:373-380. [Crossref]

10. Wagner DD, Kelley WM, Haxby JV, Heatherton TF (2016) The Dorsal Media Prefrontal Cortex Responds Preferentially to Social Interactions during Natural Viewing. J Neurosci 36: 6917-6925. [Crossref]

11. Bottari C, Gosselin N, Chen JK, Ptito A (2017) The impact of symptomatic mild traumatic brain injury on complex everyday activities and the link with alterations in cerebral functioning: Exploratory case studies. Neuropsychol Rehabil 27: 871-890. [Crossref]
12. Guo X, Song W, Chen K, Chen X, Zheng Z, et al. (2015) Impact of Frontal Lobe Function and Behavioral Changes on Health-Related Quality of Life in Patients with Parkinson's Disease: A Cross-Sectional Study from Southwest China. Eur Neurol 74 (3-4): 147-153. [Crossref]

13. Ogawa S, Lee TM, Nayak AS, Glynn P (1990) Oxygenation-sensitive contrast in magnetic resonance image of rodent brain at high magnetic fields. Magn Reson Med 14 (1):68-78. [Crossref]

14. Xiao H, Yang Y, Xi JH, Chen ZQ (2015) Structural and functional connectivity in traumatic brain injury. Neural Regen Res 10: 2062-2071. [Crossref]

15. Zhan J, Gao L, Zhou F, Kuang H, Zhao J, et al. (2015) Decreased Regional Homogeneity in Patients with Acute Mild Traumatic Brain Injury: A Resting-State fMRI Study. $J$ Nerv Ment Dis 203:786-791. [Crossref]

16. Xiong KL, Zhang JN, Zhang YL, Zhang Y, Chen H, et al. (2016) Brain functional connectivity and cognition in mild traumatic brain injury. Neuroradiology 58: 733-739. [Crossref]

17. Kinnunen KM, Greenwood R, Powell JH, Leech R, Hawkins PC, et al. (2011) White matter damage and cognitive impairment after traumatic brain injury. Brain 134: 449463. [Crossref]

18. Fujiwara E, Schwartz ML, Gao F, Black SE, Levine B (2008) Ventral frontal cortex functions and quantified MRI in traumatic brain injury. Neuropsychologia 46: 461-474. [Crossref]

19. Olsen A, Brunner JF, Indredavik EK, Finnanger TG, Vik A, et al. (2015) Altered Cognitive Control Activations after Moderate-to-Severe Traumatic Brain Injury and Their Relationship to Injury Severity and Everyday-Life Function. Cereb Cortex 25: 2170-2180. [Crossref]

20. Huang RR, Jia BH, Xie L, Ma SH, Yin JJ, et al. (2016) Spatial working memory impairment in primary onset middle-age type 2 diabetes mellitus: An ethology and BOLD-fMRI study. J Magn Reson Imaging 43: 75-87. [Crossref]

21. Gillis MM, Hampstead BM (2015) A two-part preliminary investigation of encoding-related activation changes after moderate to severe traumatic brain injury: hyperactivation, repetition suppression, and the role of the prefrontal cortex. Brain Imaging Behav 9: 801-820. [Crossref]

22. Takahashi H, Kato M, Hayashi M, Okubo Y, Takano A, et al. (2007) Memory and frontal lobe functions; possible relations with dopamine D2 receptors in the hippocampus. Neuroimage 34: 1643-1649. [Crossref]

23. Admon R, Leykin D, Lubin G, Engert V, Andrews J, et al. (2013) Stress-induced reduction in hippocampal volume and connectivity with the ventromedial prefrontal cortex are related to maladaptive responses to stressful military service. Hum Brain Mapp 34: 2808-2816. [Crossref]

24. Negoita S, Boone C, Anderson WS (2016) Directionality of Medial Prefrontal Cortex and Hippocampal Interactions Is Task-Dependent. Neurosurgery 79: N22-22N24. [Crossref]

25. Harms MP, Wang L, Csernansky JG, Barch DM (2013) Structure-function relationship of working memory activity with hippocampal and prefrontal cortex volumes. Brain Struct Funct 218:173-186. [Crossref]

26. Merkley TL, Larson MJ, Bigler ED, Good DA, Perlstein WM (2013) Structural and functional changes of the cingulate gyrus following traumatic brain injury: relation to attention and executive skills. J Int Neuropsychol Soc 19: 899-910. [Crossref]

27. Becker TM, Prat CS, Stocco A (2016) A network-level analysis of cognitive flexibility reveals a differential influence of the anterior cingulate cortex in bilinguals versus monolinguals. Neuropsychologia 85: 62-73. [Crossref]

28. Mori F, Okada KI, Nomura T, Kobayashi Y (2016) The Pedunculopontine Tegmental Nucleus as a Motor and Cognitive Interface between the Cerebellum and Basal Ganglia. Font Neuroanat 10: 109. [Crossref]

29. Yu W, Krook-Magnuson E (2015) Cognitive Collaborations: Bidirectional Functional Connectivity Between the Cerebellum and the Hippocampus. Front Syst Neurosci 9:177. [Crossref]

Copyright: (C2018 Liu Y. This is an open-access article distributed under the terms of the Creative Commons Attribution License, which permits unrestricted use, distribution, and reproduction in any medium, provided the original author and source are credited. 